\title{
Contribuciones de la filosofía fenomenológica y hermenéutica a una concepción intersubjetiva del vínculo analítico
}

\author{
André Sassenfeld J. ${ }^{1}$ \\ Santiago, Chile
}

\begin{abstract}
En este trabajo, exploro un conjunto de contribuciones de la filosofía fenomenológica y hermenéutica a una concepción intersubjetiva del vínculo analítico que se forma entre un paciente y su psicoterapeuta. Recurro a la obra de filósofos como Martin Heidegger, Maurice Merleau-Ponty, Hans-Georg Gadamer y Emmanuel Lévinas para mostrar que sus respectivas aportaciones filosóficas nos permiten concebir la relación analítica como vínculo afectivo, la situación analítica como fenómeno intersubjetivo corporeizado, el espacio analítico como espacio hermenéutico y la curvatura del espacio intersubjetivo como su dimensión ética. Comienzo esbozando la relevancia de las influencias del pensamiento filosófico en el surgimiento del movimiento relacional en el psicoanálisis contemporáneo.
\end{abstract}

Palabras clave: Fenomenologia, Hermenéutica, Vínculo analítico.

This paper explores some contributions of phenomenological and hermeneutical philosophy to an intersubjective understanding of the analytic relationship formed between a patient and her psychotherapist. I draw on the work of philosophers like Martin Heidegger, Maurice Merleau-Ponty, Hans-Georg Gadamer, and Emmanuel Lévinas to show that their respective contributions allow us to conceive the analytic relationship as an affective bond, the analytic situation as an embodied intersubjective phenomenon, analytic space as hermeneutical space, and the curvature of intersubjective space as its ethical dimension. I begin by outlining the relevance of the influence of philosophical thought on the emergence of the relational movement in contemporary psychoanalysis.

Key Words: Phenomenology, Hermeneutics, Analytic relationship.

English Title: Contributions of the phenomenological and hermeneutical philosophy to an intersubjective conception of the analytic link

\section{Cita bibliográfica / Reference citation:}

Sassenfeld J., A. (2017). Contribuciones de la filosofía fenomenológica y hermenéutica a una concepción intersubjetiva del vínculo analítico. Clínica e Investigación Relacional, 11 (3): 554580. [ISSN 1988-2939] [Recuperado de www.ceir.info ] DOI: 10.21110/19882939.2017.110307

\footnotetext{
${ }^{1}$ Psicoterapeuta analítico relacional y jungiano de adultos en práctica privada. Actualmente, es miembro del directorio internacional de IARPP y del directorio de IARPP-Chile.
} 
En este trabajo, esbozaré un conjunto de posibles contribuciones de la filosofía a una concepción intersubjetiva del vínculo psicoanalítico, concepción que ha comenzado a predominar en términos generales en el psicoanálisis contemporáneo. En particular, delinearé varios aspectos centrales que la filosofía fenomenológica y hermenéutica aporta a la comprensión de la relación analítica en el marco del psicoanálisis relacional e intersubjetivo, intentando resumir y profundizar en ideas que he presentado con anterioridad en otros contextos (Sassenfeld, 2015, 2016a, 2016b). Aunque las ideas que discutiré ciertamente tienen un sinnúmero de implicancias prácticas, no me enfocaré en una descripción clínica del vínculo analítico aunque incluiré algunas alusiones a las implicancias prácticas mencionadas. He detallado múltiples aspectos teóricos y clínicos de la relación psicoterapéutica en el marco del psicoanálisis relacional e intersubjetivo en diversas publicaciones anteriores, a las cuales el lector interesado puede recurrir (Sassenfeld, 2010a, 2012a, 2012b, 2012c, 2013a, 2014). Aquí, más bien me centraré en algunos de los fundamentos filosóficos que apuntalan y sostienen tales puntos de vista teóricos y clínicos.

Se trata de fundamentos filosóficos de relevancia central en la medida en la que el escenario psicoanalítico contemporáneo a veces ha sido caracterizado como resultado de un giro fenomenológico hacia la experiencia vivida y su comprensión (Atwood \& Stolorow, 2014 [1984]; Jordán, 2008; Leffert, 2016; Lichtenberg, Lachmann \& Fosshage, 2016; Mitchell, 1993; Reis, 1999; Sassenfeld, 2012a, 2016a, 2016b; Stern, 2015; Stern, 2004; Stolorow, Atwood \& Orange, 2002; Summers, 2013, 2015; Wachtel, 2008). En otras palabras, en el mundo psicoanalítico actual existe una cierta consciencia de cuánto el movimiento relacional le debe a la fenomenología y, tal como queda en evidencia en el trabajo de destacados teóricos relacionales e intersubjetivos (Orange, 1995, 2011; Sassenfeld, 2010b, 2012a, 2016b; Stern, 1997, 2010, 2015), también a la hermenéutica fenomenológica. Teóricos intersubjetivos como George Atwood y Robert Stolorow (2014 [1984]) han propuesto y llevado a cabo, en efecto, el desarrollo sistemático de una fenomenología psicoanalítica. Tal como señala Paul Wachtel (2008) concretizando lo que el giro fenomenológico significa, la via regia a lo inconsciente es la exploración clínica de la experiencia consciente e inmediata (véase, también, Stern, 2004).

Aunque como indiqué no me focalizaré especialmente en aspectos clínicos de la relación analítica, dicho sea de todos modos a manera de contextualización que los teóricos relacionales e intersubjetivos caracterizan el vínculo psicoanalítico a través del uso de conceptos como influencia mutua, intercambio afectivo, intimidad emocional, mutualidad asimétrica y reciprocidad (Aron, 1996; Sassenfeld, 2010a, 2012a). Con ello, dan cuenta de una conceptualización radicalmente interactiva que contrasta con aquella -con un acento más bien monádico que se desprende del llamado mito de la mente aislada (Stolorow \& Atwood, expresa. Este material es para uso científico y profesional exclusivamente y puede contener información clínica sensible. Los editores no se responsabilizan de los contenidos de los autores. Dirigir las consultas sobre derechos y autorizaciones a ceir@psicoterapiarelacional.es 
1992)- propia del psicoanálisis clásico. Es decir, se trata de una concepción bi- o multipersonal que está enmarcada por una psicología contextualista (Atwood \& Stolorow, 2014 [1984]; Coburn, 2014; Orange, Atwood \& Stolorow, 1997; Stolorow, 2011; Stolorow, Atwood \& Orange, 2002; Wachtel, 2008, 2014). Frente al trasfondo esbozado, ejes fundamentales de este trabajo son las nociones de vínculo afectivo, situación analítica, espacio hermenéutico y curvatura del espacio intersubjetivo, que discutiré a continuación. Para contextualizar la exposición que sigue, sin embargo, resulta importante mencionar antes que nada algunos puntos relativos a la relación entre filosofía y psicoanálisis relacional e intersubjetivo.

\section{Filosofía y psicoanálisis relacional e intersubjetivo}

No es este el lugar para revisar los detalles de la compleja historia de la relación entre la filosofía y la persona de Sigmund Freud (para ello, véanse Askay \& Farquhar, 2006; Orange, 2010; Sassenfeld, 2016b; y Tauber, 2010). Solo quisiera mencionar que la actitud de Freud hacia la filosofía fue de una gran ambivalencia -acentuada por su identificación con la forma de proceder característica de las ciencias naturales- y que esta ambivalencia durante mucho tiempo dificultó considerablemente la posibilidad de que los teóricos psicoanalíticos clásicos asimilaran las contribuciones que la filosofía habría podido realizar a su disciplina. (No así los fenomenólogos y los pensadores hermenéuticos en la filosofía: muchos de los grandes filósofos fenomenológicos se interesaron por el psicoanálisis -incluyendo a Jean-Paul Sartre, Maurice Merleau-Ponty, Emmanuel Lévinas y el Heidegger tardío- así como importantes filósofos hermenéuticos como Paul Ricoeur.) De acuerdo a Richard Askay y Jensen Farquhar (2006), dada su ambivalencia respecto de la filosofía, Freud y con él un sinnúmero de sus seguidores fueron incapaces de conscientizar la naturaleza de los supuestos filosóficos que subyacen a sus modelos conceptuales y por ende tampoco las implicancias prácticas de estos para el ejercicio clínico. Quedaron atrapados en el prejuicio de estar libre de prejuicios que Hans-Georg Gadamer (1969) visualiza como parte constituyente de la postura de la ciencia natural, en la cual por ejemplo el materialismo es entendido como realidad y no como supuesto filosófico. En este contexto, quizás sorprenda que, en contraste, la filosofía resultó ser una influencia fundamental en la emergencia del movimiento relacional en el psicoanálisis contemporáneo durante la década de 1980.

El surgimiento del psicoanálisis relacional e intersubjetivo está, en efecto, íntimamente ligado a la acción cuestionadora y revitalizadora de diversas influencias filosóficas sobre el psicoanálisis. De acuerdo a Jeremy Safran (2012), la (tardía) apertura de la formación psicoanalítica a candidatos que no fueran médicos a partir de la década de 1980 en los Estados Unidos estuvo vinculada a un creciente influjo de candidatos que habían 
estado expuestos previamente de modo más sistemático que los médicos a las ciencias sociales y la filosofía -en su mayoría, psicólogos. Estas emergentes nuevas voces que se fueron introduciendo de manera gradual en el discurso psicoanalítico sentían mucha mayor familiaridad con y experimentaban incluso la necesidad de una interrogación y explicitación disciplinadas de los propios supuestos filosóficos y epistemológicos -en plena congruencia con una forma particular en la que Martin Heidegger (1982 [1923]) describió la filosofía al asumir que la evitación de la pregunta por los propios supuestos corresponde a "los gritos enmascarados del miedo a la filosofía" (p. 19, cursiva del original). La circunstancia histórica y sociológica enfatizada por Safran permitió la entrada de planteamientos teóricos y clínicos alejados del racionalismo técnico propio de la medicina norteamericana al psicoanálisis y, además, la aparición de una creciente consciencia acerca de las marcadas tendencias positivistas y objetivistas subyacentes al psicoanálisis freudiano. Tal reconocimiento fue de la mano de una progresiva revisión crítica de la viabilidad de esas tendencias como fundamentos filosóficos de la teoría y práctica del psicoanálisis en el escenario general configurado por las corrientes del pensamiento propias de las últimas décadas del siglo XX.

En concreto, varios sobresalientes teóricos analíticos relacionales e intersubjetivos, incluyendo a Jessica Benjamin, Donna Orange y Robert Stolorow, tienen una formación académica filosófica adicional a su formación psicoanalítica. Mientras que Benjamin ha recibido una fuerte influencia de la filosofía de Georg Wilhelm Friedrich Hegel y su dialéctica del amo y el esclavo, Orange y Stolorow se inscriben declaradamente en la tradición fenomenológica y hermenéutica. Otros, como Stephen Mitchell, Irwin Hoffman o Donnel Stern, por mucho que no hayan tenido una formación filosófica explícita, demuestran tener conocimientos profundos de las corrientes contemporáneas principales de la filosofía -lo cual queda en evidencia con mucha claridad en sus influyentes escritos, que abordan en distintos contextos sus ideas acerca de los fundamentos filosóficos del psicoanálisis relacional. Mientras que Mitchell no se decanta de modo abierto por alguna corriente filosófica en particular, Hoffman es muy cercano al constructivismo y Stern siente afinidad con ciertos aspectos del constructivismo pero sobre todo con la hermenéutica. Desde este punto de vista, para muchos el paso fundamental que han dado los teóricos relacionales e intersubjetivos no es, en primer lugar, un paso más allá de Freud, sino más bien un paso más allá de los supuestos filosóficos cartesianos, racionalistas, cientificistas y modernos que subyacen al pensamiento de Freud y que lo determinan. Esto se ve reflejado con mucha explicitez en denominaciones que teóricos como Atwood, Stolorow y Orange han propuesto para el psicoanálisis contemporáneo: fenomenología psicoanalítica, psicoanálisis postcartesiano, etc. Sin embargo, tal paso sin lugar a dudas no puede más que conllevar la 
reformulación, relativización y ampliación de numerosas ideas freudianas originales (para un excelente resumen de este punto, véase Eagle, 2011).

Entre las corrientes filosóficas que han tenido un impacto más duradero y hondo en la emergencia del psicoanálisis relacional e intersubjetivo se encuentran especialmente la fenomenología, la hermenéutica, el pragmatismo y una variedad de filosofías postmodernas como el constructivismo y el deconstruccionismo. Todas estas corrientes tienen algo que decir acerca de la naturaleza de las relaciones humanas en general y, por consiguiente, acerca del vínculo analítico en particular. Dadas las limitaciones impuestas a este trabajo, no me es posible llevar a cabo una reseña completa de las contribuciones de todos los planteamientos filosóficos enumerados, si acaso esa tarea fuera posible de llevarse a cabo. Tal como anuncié en la introducción, me limitaré en este contexto específicamente a detallar algunas de las principales contribuciones que realizan la fenomenología y la hermenéutica. Es necesario explicitar en este sentido que el psicoanálisis relacional e intersubjetivo asume que la teorización, la práctica clínica y la escritura son inseparables de la subjetividad particular de quien teoriza, ejerce o escribe (Atwood \& Stolorow, 1993; Renik, 1993; Sassenfeld, 2012a, 2016b; Teicholz, 1999). Por lo tanto, la forma de describir en este trabajo las contribuciones fenomenológicas y hermenéuticas a la comprensión de la naturaleza de la relación analítica son, por necesidad, una versión posible de estas contribuciones, a la vez una versión teñida por mi propia subjetividad. Ya mi elección de la fenomenología y la hermenéutica de entre las demás alternativas delata mi mayor cercanía personal a sus planteamientos.

La fenomenología se ha esforzado desde sus inicios por poner al descubierto, a partir de la investigación metódica de la experiencia vivida, las diversas estructuras subyacentes que la apuntalan. En esa medida, sería equívoco considerar la fenomenología como una aproximación que se limita a explorar la experiencia subjetiva consciente. Respondiendo a la crítica de que el giro fenomenológico aleja al psicoanálisis de su histórica y valiosa dedicación a lo inconsciente, Stolorow (2010, 2011) subraya que la exploración fenomenológica nunca ha estado centrada en o limitada a la experiencia consciente como tal porque precisamente ha buscado dar cuenta de las estructuras pre-reflexivas que la sostienen y que le dan forma de manera continua fuera del foco de nuestra consciencia. Es así que teóricos intersubjetivos como Stolorow, Atwood y Orange han formulado una fenomenología característicamente psicoanalítica, que enfoca estructuras no conscientes -pre-reflexivas- de la subjetividad. Acuñan, en congruencia con su proyecto, la noción de un inconsciente pre-reflexivo que debe entenderse como un aspecto de lo inconsciente que no es excluido de modo defensivo de la consciencia (Sassenfeld, 2012a; Stolorow \& Atwood, 1992). Una diferencia de énfasis es, por cierto, que la fenomenología ha estado más interesada en estructuras pre-reflexivas 
universales de la experiencia vivida, mientras que la fenomenología psicoanalítica dedica su atención, no exclusiva pero predominantemente, a estructuras pre-reflexivas de la experiencia que cristalizan a raíz de las experiencias tempranas del individuo (véanse Sassenfeld, 2013a, 2014, 2016b y Stolorow, 2011). Se trata de lo que Stolorow, Atwood y Orange llaman principios organizadores pre-reflexivos de la experiencia.

En el marco de este trabajo, abordaré en especial algunos aspectos de la obra fenomenológica de Heidegger, Merleau-Ponty y Lévinas desde la perspectiva de sus contribuciones a una concepción intersubjetiva del vínculo analítico. Entenderé sus tres acentos como énfasis diferentes pero, a la vez, confluyentes y complementarios. La hermenéutica, por su parte, atañe directamente al psicoanálisis en cuanto se define de acuerdo a algunos teóricos como el estudio y la práctica de la interpretación, aunque en términos heideggerianos y gadamerianos este foco en la interpretación tiene que estar necesariamente enmarcado por el fenómeno más abarcador de la comprensión. La experiencia de interpretar no puede, así, desligarse del fenómeno humano de comprender. En consecuencia, no debiera sorprender que diversos teóricos psicoanalíticos hayan recurrido a sus planteamientos dado el lugar central que la interpretación ocupa en el psicoanálisis desde su nacimiento simbólico con la publicación de La interpretación de los sueños (1900) de Freud. En este contexto, me limitaré en particular a algunos aspectos centrales de la versión filosófica de la hermenéutica elaborada por Heidegger y Gadamer puesto que me parece la versión más afín con el espíritu del psicoanálisis relacional e intersubjetivo (Sassenfeld, 2010, 2012a, 2016b). Testimonio de ello ha sido su uso sistemático, ya mencionado, en el trabajo de teóricos relacionales e intersubjetivos destacados, como Donna Orange y Donnel Stern. Comenzaré por la concepción de la relación analítica como un vínculo afectivo.

\section{La relación analítica como vínculo afectivo}

En su famosa obra Ser y tiempo (1927), que marcó la filosofía europea del siglo XX de manera profunda y duradera, Heidegger se aproxima de diversas maneras a la descripción fenomenológica de la forma característica de ser de lo que denomina Dasein -ser-ahí o estar ahí ${ }^{1}$-, esto es, su idea básica acerca de lo que es para él la realidad humana primaria. En efecto, su proyecto al menos en parte gira en torno a la diferenciación de la forma particular de ser del ser humano respecto de la forma de ser de otros entes que son, como por ejemplo los objetos materiales. Busca rechazar la aplicación de categorías que son empleadas para dar cuenta de entes no humanos a la realidad humana (por ejemplo, categorías como tamaño o peso) y describir estructuras característicamente humanas en su analítica 
existencial, que intenta dar cuenta de las estructuras fundamentales que constituyen la experiencia del ser humano. Afirma que más originario que su tamaño o peso es el hecho de que el ser humano existe. De acuerdo a Heidegger, en efecto, la "esencia del Dasein yace en su existencia" (p. 42, cursiva del original), con lo cual hace alusión directa a que el existir del ser humano está indisolublemente vinculado a su presencia en un mundo. El existir del ser humano es descrito por Heidegger de modo consecuente como ser-en-el-mundo, una idea que expresa en el plano de la filosofía fenomenológica entre otras cosas lo que con anterioridad ya hemos llamado un pensamiento contextualista.

Las investigaciones fenomenológicas de Heidegger en Ser y tiempo (1927) lo llevan a reconocer entre otras cosas que la existencia del ser humano incluye la estructura prereflexiva de lo que denomina Mit-sein, esto es, ser-con, que se refiere de modo específico a que ser un ser humano equivale siempre ya a estar con y entre otros seres humanos. Fenomenólogos posteriores, como Merleau-Ponty, Sartre y Lévinas, ampliarán esta idea en dirección del concepto de intersubjetividad, que Heidegger mismo nunca ocupó debido a sus sostenidos esfuerzos por dejar atrás los conceptos de sujeto y objeto mediante la noción de Dasein. El mundo humano es siempre ya un mundo intersubjetivo, mi existencia es siempre ya una co-existencia. Incluso cuando estoy solo, la co-existencia de los otros no deja de determinar de una u otra manera la forma particular que adopta mi experiencia subjetiva. Los otros están incrustados en nuestro ser; la vinculación con otros no es, en este sentido, algo que se le añade o algo que realiza un sujeto autónomo que existe con anterioridad a entrar en vinculación con otros. Si el ser humano es, está ya vinculado con otros. Existen, no obstante, múltiples y variadas formas en las que en su experiencia vivida un individuo puede vincularse con otros. Puede experimentar relaciones superficiales y profundas, distanciadas y cercanas, simples y complejas, predecibles y caóticas.

El vínculo analítico es una forma particular en la que un individuo puede experimentar la vinculación con un otro. Es una manera específica en la que la estructura del ser-con puede manifestarse en el momento histórico en el cual vivimos. En ese contexto, sabemos que la relación psicoanalítica, como todas las relaciones psicoterapéuticas, es en algún sentido una relación artificial -¿qué otra relación humana relativamente duradera e íntima es establecida por una persona con la idea subyacente (muchas veces no consciente) de que, si la relación resulta ser constructiva, está destinada a terminarse? La teoría del apego, la investigación de infantes y la teoría psicoanalítica relacional así como las neurociencias afectivas y sociales nos muestran con mucha claridad que el ser humano está hecho, incluso biológicamente, para establecer lazos con otros seres humanos y para intentar mantenerlos a toda costa debido entre otras cosas a su valor en términos de supervivencia. En la práctica clínica, esta última parte a menudo genera importantes dificultades, tan importantes que son al menos 
uno de los aspectos que llevaron a Freud a formular ideas acerca del masoquismo y la pulsión de muerte, mientras que a Ronald Fairbairn le hicieron plantear su conocida sentencia de que la libido no busca placer, sino un objeto. En el momento de establecer una relación analítica, entonces, está implícita una separación además de una conexión y esta circunstancia es un hecho fundamental que define la forma característica de ser de la relación analítica en su dimensión del ser-con. Más allá, ¿en qué otra relación humana que gira en torno a la intimidad emocional, ésta última es planteada, al menos en términos generales, como algo unilateral?

Con todo, el carácter artificial de la relación analítica no contradice el hecho de que, para lograr alcanzar ciertos fines, esa forma de vinculación puede resultar ser sumamente útil o incluso necesaria. El psicoanálisis relacional e intersubjetivo supone que un proceso analítico exitoso está basado en, y por ende requiere del establecimiento de un vínculo afectivo entre paciente y terapeuta. Esto significa, por un lado, que se trata de un lazo que en primer lugar pasa por la emocionalidad que se desarrolla entre quienes participan de él y, por otro lado, que el foco de la relación es la vida emocional del paciente y -quizás un tanto más implícita o delimitadamente- del psicoterapeuta. En congruencia con esa suposición, una aproximación analítica intersubjetiva busca desplegar actitudes del psicoterapeuta que faciliten y promuevan un vínculo de tal naturaleza, incluyendo actitudes como la amabilidad, la empatía, la disponibilidad emocional, la autenticidad, la compasión y otras (BCPSG, 2010; Lichtenberg, Lachmann \& Fosshage, 1996; Orange, 1995, 2011; Sassenfeld, 2012a; Stern, 2004). Es indispensable promover, y a menudo restablecer, tal vínculo porque no puede darse por supuesto que éste emergerá o se sostendrá en el tiempo por sí solo, una lección que hemos aprendido al menos desde que Heinz Kohut formulara la psicología del self durante la década de 1970 (véase Sassenfeld, 2006). Desde el punto de vista fenomenológico, es significativo que los teóricos relacionales e intersubjetivos enfaticen así la importancia de una cierta forma de estar en relación con el otro. Esto no quiere decir que en el vínculo analítico no se produzcan momentos marcados por fallas empáticas, inautenticidades, impasses, ausencias de compasión o amabilidad o escenificaciones "colisiones psicoanalíticas" (Bromberg, 2006; Slochower, 2014 [2006]). Sin embargo, tales experiencias relacionales ocurren en el caso ideal en el seno de un vínculo afectivo que, si es efectivamente afectivo y firme, podrá soportarlas y en el mejor de los casos permitir su elaboración.

Heidegger (1927) describe como otra estructura pre-reflexiva de la experiencia característica del ser humano lo que, con un neologismo alemán, denomina Befindlichkeit. Befindlichkeit quiere decir algo así como la forma-en-la-que-uno-se-encuentra-en-unasituación, implicando que el individuo siempre se encuentra ya de una cierta manera en la 
situación en la que está. Stolorow (2011) indica que, a su parecer, este término utilizado por Heidegger da cuenta de la estructura pre-reflexiva de la afectividad. Por consiguiente, la noción heideggeriana destaca que el ser humano se encuentra siempre ya percibiéndose, sintiéndose y experimentándose afectivamente a sí mismo de cierto modo en cualquier situación que enfrente. Desde esta perspectiva, los analistas relacionales e intersubjetivos buscan, porque consideran necesaria, por así decir, una determinada atmósfera emocional en la relación con el paciente, una determinada manera afectiva de estar juntos en la situación analítica y de relacionarse el uno con el otro. Aunque esta idea le es ajena a la filosofía fenomenológica, teórica y clínicamente los terapeutas relacionales e intersubjetivos han aprendido que ciertas atmósferas emocionales pueden facilitar o entorpecer la exploración conjunta de la experiencia subjetiva y, con ello, la posibilidad de articular y dar forma consciente y reflexiva a los aspectos y las estructuras inconscientes particulares que determinan la experiencia vivida del paciente. En la actualidad, gracias a las significativas contribuciones de la teoría del apego, existe un cierto consenso respecto de que la variable principal de una atmósfera afectiva clínicamente constructiva es la experiencia mutua de seguridad, por mucho que, para que sea posible la elaboración analítica de los aspectos traumáticos y disociados de la experiencia, siguiendo la formulación de Philip Bromberg (2006), el vínculo terapéutico tiene que ser seguro, pero no demasiado seguro.

La noción de Befindlichkeit elaborada por Heidegger subraya la indisoluble ligazón que existe entre la afectividad y la situación en la que alguien se encuentra, dando lugar a una perspectiva contextual sobre la experiencia afectiva que es plenamente congruente con el contextualismo ya mencionado de las teorías analíticas relacionales e intersubjetivas. En otras palabras, la forma en la que un individuo se siente de modo habitual o puntual no es comprensible al margen de los contextos relacionales en los que se mueve de modo habitual o puntual, un principio que es intrínseco al psicoanálisis relacional e intersubjetivo. La manera en la que un paciente o un terapeuta dado se siente en o después de una sesión tiene directa relación con las características que la situación relacional, en la que ha participado, adopta. Esto no significa que el pasado de cada uno y la forma en la que este se actualiza en el presente es irrelevante o inexistente; más bien, significa que, aún frente a la intensa presencia del pasado en el presente, que se manifiesta por medio de la actividad continua de los principios organizadores de la experiencia, la forma en la que esa circunstancia es manejada en el vínculo analítico marca tremendas diferencias en términos de la experiencia subjetiva e intersubjetiva de paciente y analista por igual. En términos del vínculo psicoanalítico, con lo que he señalado hasta aquí se configura una tríada de vinculación (Mitsein o estar-con), afectividad (Befindlichkeit o estar-cómo) y situación, a partir de algunas contribuciones importantes de la fenomenología que hemos destacado en lo que antecede. 
Dada su relevancia particular para la temática de este trabajo, examinaré el tercer elemento de la situación en la próxima sección con mayor detención.

La situación analítica: un fenómeno intersubjetivo corporeizado

La descripción que Heidegger (1927) formula de la existencia humana como Dasein da cuenta de que ser un ser humano implica estar situado; el "ahí" (Da) implica estar ubicado concretamente en un lugar físico, pero también implica al mismo tiempo estar situado más ampliamente en lo que Heidegger describe como un mundo con horizontes característicos y lo que Gadamer (1960) llama tradición y prejuicio². Se trata de horizontes que son siempre al mismo tiempo históricos, lingüísticos, culturales, sociales, psicológicos y emocionales. Ser un ser humano significa existir en un mundo, estar "arrojado" a un mundo que nos precede y que está siempre ya situado en términos históricos. Ser un ser humano quiere decir, por lo tanto, estar siempre ya situado y además encontrarse siempre ya en una situación. La famosa expresión ser-en-el-mundo empleada por Heidegger remite, en el sentido que le hemos atribuido con anterioridad, a aún otra estructura pre-reflexiva del ser. Nuevamente, la situación analítica es una forma particular de situación en la que el individuo puede encontrarse o una modalidad específica de estar-ahí que una persona puede experimentar en nuestra época histórica actual. Distintiva de la situación analítica es su naturaleza intersubjetiva por definición, en ella participan y se encuentran (al menos) dos mundos de experiencia organizados de modos diferentes, esto es, dos mundos con horizontes delimitadores distintos (Stolorow, Atwood \& Orange, 2002).

Es fundamentalmente Merleau-Ponty (1945) quien desarrolla la descripción fenomenológica de la dimensión física y corporal del estar-situado y lo hace, de manera significativa para el psicoanálisis intersubjetivo, en íntima ligazón con la intersubjetividad. Su pensamiento subraya que existe una inter-corporalidad como característica definitoria de la existencia humana, una matriz intersubjetiva corporeizada en el seno de la cual emerge el sujeto. Para Merleau-Ponty, por ende, es esta inter-corporalidad basal la que deja al descubierto la vinculación constitutiva entre un sujeto y otros sujetos en cuanto precede a la experiencia reflexiva y consciente de uno mismo como sujeto separado de otros sujetos. Esta forma de vinculación tiene que ser visualizada como vinculación entre sujetos corporeizados y que, por consiguiente, no solo tiene materialidad, sino también espacialidad. Desde ese punto de vista, es importante enfatizar -por obvio que pueda parecer una vez enunciado- 
que la situación analítica es intrínsecamente una situación espacial. A veces, este aspecto se mantiene en un segundo plano de la consciencia de los psicoterapeutas; pero, por ejemplo, es necesario reconocer que la situación analítica tradicional está en gran medida definida en términos espaciales en cuanto ubica a terapeuta y paciente en una cierta disposición espacial uno respecto del otro. Es tan relevante esta configuración espacial, que implica tanto una fundamental asimetría de las posturas corporales (analista sentado, paciente recostado) como la ausencia de contacto visual y, así, es una disposición espacial que implica un conjunto de simultáneas restricciones y posibilidades relacionales. En otro lugar, intenté mostrar que el setting psicoanalítico clásico involucra una verdadera intervención corporal en términos de espacialidad (Sassenfeld, 2007).

Por otro lado, la inter-corporalidad es el fundamento necesario de la posibilidad de comprender al otro ya que, para Merleau-Ponty (1945), la comprensión del otro en el plano de la experiencia vivida es un fenómeno pre-reflexivo que tiene lugar de modo primario en el plano corporal (véase, también, Sassenfeld, 2012c, 2013a, 2013b). Merleau-Ponty afirma: "existe entre este mi cuerpo fenoménico y el del otro tal como lo veo desde afuera una vinculación interna, que provoca que el otro aparezca como la completación de un sistema" (p. 410), una idea que transformé en la noción de un sistema intersubjetivo corporeizado caracterizado por una influencia mutua corporeizada (Sassenfeld, 2012b, 2012c, 2013a, 2014). Agrega: "es precisamente mi cuerpo el que percibe el cuerpo del otro y descubre en ese cuerpo una milagrosa extensión de mis propias intenciones, una manera familiar de tratar con el mundo" (p. 412). He llamado al proceso descrito aquí por Merleau-Ponty con anterioridad lectura corporal implícita que, al interactuar de modo directo con alguien, se convierte en lectura corporal implícita recíproca (Sassenfeld, 2008, 2010c, 2013c). Esta perspectiva, que Merleau-Ponty articula apoyándose en ideas de Edmund Husserl, ha sido tan influyente que ha sido retomada por parte de investigadores neurocientíficos destacados como Vittorio Gallese (2009). La comprensión explícita y consciente del otro es, en ese sentido, una experiencia secundaria derivada de la comprensión más basal del otro que caracteriza la inter-corporalidad. Así, desde el punto de vista de las contribuciones de Merleau-Ponty, es posible entender la situación analítica como situación intersubjetiva corporeizada (Sassenfeld, 2012b, 2012c, 2013a, 2014, 2016b).

Esta descripción fenomenológica de la situación analítica se enlaza de manera directa con el papel esencial que cada vez más teóricos analíticos relacionales e intersubjetivos han atribuido a la dimensión del intercambio no-verbal en el proceso analítico. Tal como he buscado mostrar en otro lugar (Sassenfeld, 2007), la dimensión de la corporalidad ha sido una temática conflictiva en el psicoanálisis clásico (véase, también, Sletvold, 2014). Owen Renik (1993) enfatiza, en este mismo sentido, que el pensamiento freudiano acerca de la 
acción y la corporalidad está basado en premisas muy cuestionables hoy en día, que suponen que el destino alternativo de un impulso es su representación mental o su descarga a través del comportamiento -como si cognición y acción fueran fenómenos mutuamente excluyentes. El pensamiento freudiano dio lugar al concepto del acting out que, para numerosos teóricos relacionales e intersubjetivos, tiene connotaciones enjuiciadoras y peyorativas. El intento constructivo de la teoría relacional ha sido intencionar una transición hacia el concepto de enactment, que busca entender de forma diferente el lugar tan desvalorizado de la acción y el comportamiento en el psicoanálisis (Sassenfeld, 2010d, 2012a). En ese contexto, las nociones fenomenológicas formuladas por Merleau-Ponty ofrecen un marco filosófico relevante para ubicar el énfasis psicoanalítico contemporáneo en la dimensión no-verbal de la interacción psicoterapéutica y en su profundo potencial de cambio y transformación (BCPSG, 2010). Si somos, siguiendo a Merleau-Ponty, seres corporales e intersubjetivos, el proceso analítico exitoso no puede más que transformarnos en términos al mismo tiempo corporeizados y relacionales, en el seno de un sistema intersubjetivo corporeizado que tiene planos conscientes e inconscientes.

Retomando desde aún otro ángulo un aspecto ya mencionado, que Merleau-Ponty (1945) recoge de Heidegger y que elabora en sus propios planteamientos, es la noción de espacialidad. Al concebir el sujeto como sujeto corporeizado, Merleau-Ponty no puede evitar abordar la problemática de la existencia en el espacio y de la experiencia del espacio. La corporalidad de la existencia humana no puede desligarse de su espacialidad: si ser un ser humano significa ser un cuerpo, implica a la vez ocupar siempre ya un lugar en el espacio y habitar, literalmente, siempre ya una determinada perspectiva respecto del mundo. En otras palabras, el cuerpo es subjetividad puesto que equivale a encontrarse en una perspectiva particular, diferente de aquella que ocupan otros en un momento dado. Frente a este trasfondo, no solo puede hablarse de una situación analítica, sino también además de un espacio analítico. En otras palabras, al ser la espacialidad una estructura pre-reflexiva del ser, existe una forma particular de estar en el espacio que llamamos analítica. Discutiré este punto con mayor detalle en la próxima sección. Ahora bien, ¿qué convierte una situación interpersonal cualquiera en una situación analítica? Y, ¿qué transforma una determinada experiencia del espacio en una experiencia analítica del espacio? Existen múltiples maneras de responder estas interrogantes; para muchos, por ejemplo, el uso del encuadre analítico convierte una situación intersubjetiva en un sentido más general en una situación específicamente analítica. No obstante, tales respuestas no son una preocupación propia de la filosofía y, por ende, no nos detendremos en este contexto en dilucidarlas con mayor atención en la medida en la que trascienden los objetivos de este trabajo. 


\section{El espacio intersubjetivo como espacio hermenéutico}

Heidegger (1927) destacó que el ser humano, en su experiencia vivida, no se mueve en un espacio tridimensional afectivamente neutro, como aquel que las ciencias naturales retratan y tratan como realidad. Más bien, el ser humano se mueve en un mundo en el cual experimenta una fundamental familiaridad con las cosas, un mundo llamado por Husserl (1936) mundo de la vida (Lebenswelt). En palabras de Merleau-Ponty (1945), la espacialidad humana no es una espacialidad de la posición de un objeto en el espacio, sino la espacialidad de una persona en una situación. Sabemos, de una u otra manera y en cierta medida, movernos en el mundo de la vida y tratar con las situaciones que surgen en éste porque estamos familiarizados siempre ya con él. La familiaridad vivida con el mundo forma parte intrínseca de quiénes somos. Para Heidegger, esta circunstancia muestra que el mundo vivido no es en primer lugar un espacio propiamente "físico" en el sentido científico natural, sino un espacio cuya materialidad es inseparable de lo que significa para el individuo que se encuentra en él. En efecto, fenomenológicamente mundo y significado se corresponden, son indivisibles y siempre coinciden en nuestra experiencia cotidiana pre-reflexiva y reflexiva.

Desde este punto de vista, Heidegger describe la comprensión como estructura prereflexiva del ser, asumiendo que ser un ser humano equivale a estar siempre ya en una cierta comprensión del mundo o, con palabras de Gadamer, estar ubicado en una cierta constelación de prejuicios y de la tradición que nos permite aprehender nuestra realidad en cierta medida y en cierta forma. Dicho de otro modo, el ser humano es un ser hermenéutico, esto es, un ser que comprende e interpreta tanto el mundo como su propia experiencia, un animal que se interpreta a sí mismo, como asevera Charles Taylor (1985). Al igual que la noción de inter-corporalidad formulada por Merleau-Ponty, la noción hermenéutica de comprensión que describe Heidegger y que será desarrollada de modo profundo y extenso por Gadamer no hace referencia de modo primario a la posibilidad consciente y explícita de comprender algo, sino a esa familiaridad cotidiana con las cosas que, para ser experimentada, presupone una comprensión del mundo. Para subir con éxito una escalera, requiero de una comprensión implícita acerca de las posibilidades de movimiento de mi cuerpo, acerca de la fuerza de gravedad, acerca de las distancias, etc. Toda aquella comprensión se plasma y está en juego cuando, efectivamente, subo la escalera que se encuentra frente a mí, pero no se trata de una comprensión que por lo común sea explícita y reflexiva.

En la obra de Merleau-Ponty (1945), la forma de comprender recién descrita está vinculada a la noción de hábito, un saber hacer determinadas cosas en el mundo y en el espacio y con otros que parece ser inherente al cuerpo (Sassenfeld, 2013a, 2014, 2016b). 
Merleau-Ponty escribe: "es el cuerpo el que 'capta' y 'entiende' el movimiento. La adquisición de un hábito es, en efecto, la comprensión de un significado, pero es la comprensión de un significado motriz" (p. 165). Expresa, más allá, que "el proceso de comprender un significado es realizado por el cuerpo" (p. 177) y que mi "cuerpo es el tejido en el cual todos los objetos están entrelazados y es, al menos en relación con el mundo percibido, la herramienta general de mi 'comprender'"' (p. 273). En consecuencia, para él, un hábito es lo que hace al sujeto sentirse parte del mundo, lo que lo inserta en un mundo compartido con otros sujetos corporeizados. Pienso que la noción merleaupontiana de hábito es de significación para el psicoanálisis intersubjetivo porque es un precursor importante de conceptos esenciales como la memoria procedural y el conocimiento relacional implícito (BCPSG, 2010; Sassenfeld, 2013b). El Boston Change Process Study Group, por ejemplo, ha subrayado que el conocimiento relacional implícito abarca todo aquello que sabemos hacer de manera implícita -es decir, no consciente o pre-reflexiva- con y respecto de los demás. En trabajos anteriores, he interpretado la noción fenomenológica del hábito adicionalmente como noción que da cuenta del aspecto sensoriomotriz de lo que la teoría psicoanalítica de los sistemas intersubjetivos conceptualiza como principios organizadores de la experiencia (Sassenfeld, 2013a, 2014, 2016b).

Considero que una forma útil de conceptualizar lo que Heidegger y Gadamer subrayan, incorporando las contribuciones ya mencionadas de Merleau-Ponty, es afirmar que el ser humano se encuentra siempre ya en un espacio hermenéutico (Figal, 2006; Sassenfeld, 2015, 2016a, 2016b). En otras palabras, el ser humano experimenta de modo continuo y habita cotidianamente un espacio vivido que es siempre ya materialidad significativa y significación materializada y que, por lo tanto, es vivenciado siempre ya con comprensión pre-reflexiva y que a menudo se vuelve objeto de interpretación explícita. Lo que nos rodea es siempre ya comprensión pre-reflexiva y muchas veces necesita ser interpretado en términos reflexivos. En el existir humano, co-existen siempre ya un mundo necesitado de comprensión y un ser humano necesitado de comprenderlo (Angehrn, 2004; Sassenfeld, 2016b). Desde el punto de vista fenomenológico, tal vez podría denominarse esta circunstancia intencionalidad hermenéutica: el mundo humano es un espacio hermenéutico en el cual necesitamos comprender e interpretar lo que nos rodea porque lo que nos rodea nos interpela. El espacio hermenéutico es un espacio concreto tal como ya pudimos evidenciar al discutir la corporalidad y la espacialidad y, al mismo tiempo, un espacio de significación y de significaciones potenciales. A mi parecer, es para este espacio que Heidegger acuña, en el fondo, la noción de mundo. En ese sentido, la noción del espacio hermenéutico -acuñada en primer lugar por el fenomenólogo alemán contemporáneo Günter Figal (2006)- intenta especificar lo que Heidegger quiso decir cuando articuló su 
noción de mundo. Dada la extensión de esta sección, podrá notarse que en mi forma de pensar, la noción del espacio hermenéutico es, puesto que a mi parecer engloba las nociones discutidas en las secciones previas, la de mayor relevancia desde mi punto de vista.

Dado que la significación en el sentido más amplio del término -el mundo- es un fenómeno que precede al individuo, al cual éste se encuentra arrojado y en cuyo seno siempre ya se encuentra, el espacio hermenéutico es necesariamente un espacio de carácter intersubjetivo. Recordemos, en este contexto, la estructura pre-reflexiva del ser-con que Heidegger (1927) describió. Posiblemente, el mejor ejemplo de ello es el lenguaje, en y a través del cual el ser humano es capaz de articular explícitamente significaciones y comprensiones. Tanto Heidegger como Gadamer dedicaron grandes esfuerzos a estudiar la naturaleza del lenguaje y del habla y, en especial, Gadamer (1960) llegó a la conclusión de que el ser que puede ser comprendido es lenguaje. Esta declaración central de su obra capital Verdad y método (1960) ha sido en sí misma objeto de diversas interpretaciones (Grondin, 2001; Rorty, 2001). En una forma posible de interpretar esta aseveración de Gadamer, por un lado el ser humano no puede, en realidad, moverse fuera del lenguaje: también el lenguaje es una estructura pre-reflexiva del ser. Esto no quiere decir que el individuo no pueda atravesar ciertas experiencias que, en términos vivenciales, generan la impresión subjetiva de no ser de carácter verbal o lingüístico. Solo implica que hasta esas experiencias están pre-reflexivamente determinadas por el lenguaje ya que éste contribuye de modo intrínseco a dar forma a la experiencia subjetiva. Por otro lado, la aseveración de Gadamer enfatiza que la comprensión está ligada íntimamente al lenguaje. Sea como sea, la existencia del lenguaje es, en términos de la fenomenología y la hermenéutica, sinónimo de la existencia de otros ${ }^{3}$.

A mi parecer, existen al menos dos formas fundamentales en las que el ser humano puede encontrarse o moverse en el espacio hermenéutico, dos formas básicas que no son mutuamente excluyentes (Sassenfeld, 2015, 2016a, 2016b). Tal como nos enseña la fenomenología en general, la forma más habitual de movernos en el espacio hermenéutico en cuanto mundo de la vida es hacerlo de manera pre-reflexiva e implícita. Como apunté con anterioridad, el individuo en lo cotidiano lidia con el mundo basado en una comprensión intrínseca simultánea de su materialidad y del significado de las cosas, a esa circunstancia se debe que hablemos del mundo como espacio hermenéutico. Cuando suena el timbre en mi consulta, me levanto y voy a abrir la puerta. En un plano habitualmente aún más prereflexivo, tal como la investigación de infantes ha mostrado de manera magistral, percibo cuando es mi turno de hablar a la hora de interactuar con mi paciente e intervengo en consecuencia. Actúo sabiendo lo que tales situaciones significan, no hace falta que escoja entre múltiples alternativas de significación de manera consciente y deliberada. Me ajusto a 
lo que a menudo fuera de mi consciencia focal percibo que está transcurriendo, por mucho que con frecuencia no ocurra de modo perfecto. Estas experiencias guardan por lo común una relación directa con la forma en la que vivenciamos la inmediatez del momento presente en la medida en la que se despliega y buscamos lidiar con lo que este trae consigo (Stern, 2004).

Por otro lado, existe una forma más bien explícita de encontrarnos en el espacio hermenéutico. Siguiendo a Gadamer (1958), el punto de partida de esa experiencia es una especie de irritación que se expresa en la percepción de algo como "ajeno" -algo destaca, sobresale, y nos interpela, no logramos seguir simplemente adelante sin tomarlo en consideración. A veces nos sorprende porque no es algo que esperábamos, otras veces nos puede molestar porque lo sentimos como una interrupción. Sea como sea, se nos muestra como algo ajeno, como algo que no encaja, y en consecuencia como algo necesitado de comprensión y, con ello, encamina un esfuerzo consciente por comprender. Mi paciente usa una palabra que me extraña y siento la necesidad de pedirle una aclaración con la finalidad de entender mejor a qué se refiere. Leo un trabajo psicoanalítico y de pronto una frase me hace detenerme para cuestionarme si en realidad la he comprendido y me lleva a reflexionar con mayor detención. Así, la comprensión (o bien su ausencia o dificultad) se convierte en experiencia consciente $y$, en esa medida, el sujeto no solo comprende (o no), sino que su comprender pasa a constituir la articulación explícita de una interpretación. En otras palabras, la hermenéutica denomina interpretación a la explicitación y explicitez de un acto de comprensión (Sassenfeld, 2016b). Desde esta perspectiva, el ser humano existe en el espacio hermenéutico al mismo tiempo comprendiendo pre-reflexivamente e interpretando reflexivamente.

En ambas formas de estar en el espacio hermenéutico, como mencioné antes, el individuo está siempre ya situado ocupando una determinada perspectiva. Gadamer (1960) hace referencia a ese inevitable y necesario estar situado como prejuicio, una noción que, tal como detallé con anterioridad, Gadamer desea rehabilitar con la finalidad de desprenderla de las connotaciones negativas históricas que ha adquirido desde la Ilustración (Sassenfeld, 2010b, 2012a, 2016b). Tanto mi pertenencia a una tradición histórica, social y cultural como mis experiencias personales previas configuran la estructura específica de mis prejuicios, que en un sentido radical da cuenta de mi posibilidad de apertura al mundo; esto es, de acuerdo a Gadamer, sin prejuicio y pertenencia a una tradición no podría comprender el mundo y las cosas. Prejuicio y tradición son, en sí mismas, una especie de comprensión condensada del mundo. En términos psicoanalíticos, dado el énfasis del psicoanálisis en la dimensión biográfica por encima del énfasis muchas veces más histórico y colectivo de la filosofía, prejuicio y tradición incluyen en especial nuestros hábitos relacionales corporeizados -que 
hemos empezado a llamar conocimiento relacional implícito- y nuestros principios organizadores de la experiencia. En términos psicoanalíticos, estamos hablando de que, siguiendo a Orange (1995), el sujeto es un organizador activo de su experiencia. Por supuesto, lo dicho no implica que prejuicio y tradición no puedan a la vez obstaculizar o distorsionar mi comprensión del mundo. El prejuicio, sobre todo cuando es inflexible e inconsciente, nos dificulta abrirnos a formas alternativas de comprender el mundo y nuestra experiencia.

Lo que he afirmado en esta sección nos permite concebir el espacio analítico como una cierta modalidad del espacio hermenéutico. Teniendo una materialidad propia (ubicación de la consulta, dimensiones y características de la sala de atención, decoración y arreglo de la sala de atención, etc.), la situación analítica se constituye como un espacio hermenéutico especial en la medida en la que el vínculo entre paciente y psicoterapeuta y su trabajo conjunto gira en torno a las significaciones existentes y potenciales de la experiencia subjetiva. El prejuicio y la interpretación, en los planos implícito y explícito respectivamente, estructuran el espacio hermenéutico $y$, al hacerlo, confieren formas particulares a la experiencia. Reducen la potencialidad abierta de significados posibles al situar a quien o a quienes buscan entender en determinadas perspectivas y, con ello, delimitan sus horizontes de experiencia. Sin tales posicionamientos, no existiría comprensión en el sentido hermenéutico del término; no obstante, como ya señalé, los mismos posicionamientos que posibilitan comprensión pueden a la vez dificultarla e incluso imposibilitarla. El espacio hermenéutico se transforma en un espacio analítico cuando abre la posibilidad de reflexionar interpretativamente en conjunto sobre la forma particular en la cual el prejuicio del paciente, del psicoterapeuta y ambos en combinación estructuran el espacio hermenéutico, dando lugar a configuraciones habituales más o menos flexibles de la experiencia subjetiva e intersubjetiva.

Para Gadamer (1960), lo recién descrito implica poner en juego los propios prejuicios y arriesgarlos, tanto para tomar consciencia de ellos como para volver posible su eventual corrección, ampliación o transformación. Ello significa, en contraste con el acento característico del psicoanálisis freudiano, que el terapeuta intersubjetivo tiene que experimentar una relativa libertad relacional (Stern, 2015) a la hora de poner en palabras lo que está pensando y sintiendo para contribuir con relativa continuidad a la reflexión conjunta con su paciente (Sassenfeld, 2016b); y subrayo su relativa libertad y continuidad porque, por supuesto, la participación verbal activa de cualquier psicoterapeuta está enmarcada por la atención sistemática al despliegue del proceso afectivo y psíquico de cada paciente. Más allá, la reflexión en busca de comprensión emocional transformadora, compartida por paciente y psicoterapeuta, no debe visualizarse nunca primariamente como ejercicio de carácter 
intelectual, sino más bien como la experiencia afectiva y relacional que conforma la esencia del proceso analítico desde el punto de vista del psicoanálisis intersubjetivo (Orange, 1995). Esta idea encaja muy bien con lo que, en una sección previa, afirmé respecto de la noción heideggeriana de Befindlichkeit y la relación analítica como vínculo afectivo. Así, el espacio hermenéutico analítico es la matriz que posibilita el cumplimiento gradual de una de las metas centrales de la psicoterapia intersubjetiva: desplegar, iluminar en términos interpretativos y transformar mundos subjetivos de experiencia (Buirski \& Haglund, 2001; Sassenfeld, 2012a, 2016b; Stolorow, Brandchaft \& Atwood, 1987).

Desde el punto de vista de cómo el psicoterapeuta estructura el espacio hermenéutico es esencial hacer alusión a que los conceptos analíticos que cada terapeuta maneja son una dimensión central de esa estructuración. Su posicionamiento teórico define, en gran medida, su experiencia del espacio analítico y las posibilidades de significado y sentido de los fenómenos que surgen en él. Por ejemplo, cuando Kohut (1977, 1984) concibe un conjunto significativo de reacciones agresivas de parte del paciente como ira narcisista cuya función psíquica es restablecer -aunque de forma precaria- la cohesión de un self que está fragmentando y no como manifestaciones de una destructividad intrínseca (como, posiblemente, las podría concebir un terapeuta kleiniano o kernbergeriano), se posiciona de cierto modo en el espacio hermenéutico. Es decir, hace uso de manera relativamente consciente de un prejuicio que implica estructurar el espacio hermenéutico al adoptar en este una determinada perspectiva y delimitar el rango de las significaciones de ciertos fenómenos; escribo "relativamente consciente" porque, con el paso del tiempo, muchos de los conceptos que un terapeuta emplea en su labor cotidiana pasan a estructurar el espacio hermenéutico de manera pre-reflexiva al convertirse en modalidades habituales de entender las cosas. La concepción mencionada de Kohut que se coloqué como ejemplo permite al terapeuta comprender algo -una respuesta agresiva- como algo -un intento subjetivo desesperado de cohesionar un self en fragmentación y, así, regular la angustia asociada al estado de fragmentación.

Comprender algo como algo es una estructura básica que Heidegger (1927) llamó el como hermenéutico (hermeneutisches Als) (véase Sassenfeld, 2016b). El como hermenéutico significa que, cuando comprendemos, siempre comprendemos lo que comprendemos como algo. Cuando escucho un sueño relatado por un paciente, lo escucho con atención porque lo entiendo como manifestación significativa de lo inconsciente y eso me parece relevante en términos analíticos. Cuando presto atención a la forma particular en la que un paciente entra a mi consulta entiendo esa forma como expresión de su personalidad y eso me resulta importante. Desde este punto de vista, todos los conceptos analíticos son, en esencia, comos-hermenéuticos o interpretaciones de lo que ocurre que al mismo tiempo posibilitan 
y restringen nuestra comprensión clínica. Si entiendo un sueño como manifestación de lo inconsciente, no lo puedo entender como algo irrelevante y ese hecho ya me lleva hacia una dirección definida hacia la que otras alternativas no me hubiesen conducido; como ya he afirmado, un prejuicio de modo simultáneo posibilita y delimita nuestra comprensión. Muchas dificultades terapéuticas están vinculadas con la circunstancia de que un terapeuta utiliza ciertos conceptos de forma inflexible rigidizando la libertad de movimiento en el espacio hermenéutico y, con ello, genera actitudes reduccionistas respecto de la experiencia en cuestión. El uso rígido de los conceptos analíticos cierra la posibilidad de reflexionar de modo abierto sobre los significados potenciales de una experiencia.

Entonces, las dos formas descritas de cómo un sujeto puede estructurar el espacio hermenéutico -consciente e inconsciente- pueden, a su vez, operar cada una de dos maneras diferentes. Sea como prejuicio o principio organizador de la experiencia o como interpretación, la forma en la que un sujeto estructura el espacio hermenéutico puede ser flexible y adaptativa o inflexible y rígida. Como paralelos en la teoría relacional, Donnel Stern (1997, 2010) hace alusión a que la disociación puede impedir de modo sostenido y por ende inflexible la formulación de determinados significados de la experiencia, mientras que Orange (1995) -y también Stolorow y sus colaboradores (Orange, Atwood \& Stolorow, 1997; Stolorow \& Atwood, 1992; Stolorow, Atwood \& Orange, 2002)- señala que un principio organizador puede dar forma a la experiencia de modo rígido e inconsciente o de modo flexible y consciente. Un principio organizador de la experiencia que actúa sin consciencia impide el surgimiento de experiencias distintas de las habituales y, al mismo tiempo, tiende a generar experiencias con formas muy similares. Estructurar el espacio hermenéutico de manera inconsciente e inflexible restringe de modo innecesario lo que Stolorow, Atwood y Orange (2002) denominan horizontes de la experiencia y, con ello, literalmente empobrecen el mundo subjetivo. El rango de las posibilidades de experiencia se estrecha y estereotipa. El individuo se ve encerrado en un mundo que ya es conocido, que atraviesa escasas variaciones y que, por lo común, no es vivido como satisfactorio o que incluso produce sufrimiento. Los significados y el sentido de muchas experiencias se mantienen invariables y no le permiten al sujeto reconocer y asimilar experiencias novedosas que podrían desconfirmar sus prejuicios. En otras palabras, el sujeto pierde libertad de movimiento por el espacio hermenéutico y su mundo de la vida, siendo la libertad una de las dimensiones fundamentales que, siguiendo a Figal (2006), constituyen el espacio hermenéutico. Mientras mayor libertad vivida de movimiento existe en el espacio hermenéutico, mayor es nuestra apertura a, y exploración, de diversas posibilidades de significado.

Quiero ejemplificar lo que he desarrollado en esta sección. Un paciente de mediana edad había experimentado de modo crónico una falta de disponibilidad afectiva de parte de 
su figura materna a lo largo de gran parte de su infancia y niñez debido a una depresión nunca tratada de ella. Estas experiencias dieron lugar a un prejuicio o convicción emocional (Orange, 1995) que le hacía comprender una diversidad de situaciones relacionales como abandonos, pérdidas y decepciones, sin que fuera consciente de los orígenes de sus sentimientos habituales en su historia de vida -su "tradición" personal. Este prejuicio perteneciente a su tradición, tal como muestra Gadamer, le permitía efectivamente comprender muchas situaciones que vivenciaba; sin embargo, le era casi imposible sentir que podía también estar equivocado y que por ende podían existir otras alternativas de significado de las mismas situaciones, predominando el supuesto tácito de que estaba en lo correcto. En general, las explicaciones que los demás en ocasiones intentaban proporcionarle de por qué habían actuado como habían actuado y su insistencia en que no se trataba de abandonos eran interpretadas por parte de este paciente como justificaciones o incluso como mentiras. El mundo de experiencia de mi paciente era un mundo restringido en el cual lo acostumbrado eran el abandono, la pérdida y la decepción, sin que fuera posible tener por ejemplo la experiencia de que los otros en muchas situaciones tienen necesidades o preocupaciones propias o que, sin intenciones negativas o egoístas, no siempre logran reconocer lo que a él le ocurre o lo que necesita. Sesión tras sesión presentaba más y más relatos pasados y actuales en los cuales se repetía la experiencia descrita con formas casi idénticas. Estábamos tanto frente a un principio organizador de la experiencia que actuaba de modo inconsciente e inflexible como frente a una cierta interpretación consciente pero rígida de las cosas, dos aspectos que tienden a co-existir en cada paciente. Notoria y a la vez dolorosa era la pobreza de sus posibilidades de experiencia en las relaciones afectivas con los demás, que era reflejo de la ausencia de libertad que vivenciaba una y otra vez.

Siguiendo a Gadamer (1960), un proceso de reflexión como el descrito en esta sección en el contexto de un proceso analítico transcurre en el seno del lenguaje y, para él, la naturaleza del lenguaje es dialógica. En una interpretación apoyada en cómo Heidegger tiende a circunscribir el significado del término de origen griego logos, podría tal vez aseverarse que el dia-logos es aquello que acontece y se revela por medio del habla, que en términos gadamerianos es siempre un hablar con otro (en ocasiones, o a menudo, el otro es uno mismo) (Sassenfeld, 2010b, 2012a, 2016b). Por lo tanto, la forma propiamente analítica de estar en el espacio hermenéutico corresponde a aquella de cuestionar, jugar con y reorganizar reflexivamente significados implícitos y explícitos, inconscientes y conscientes, por medio de un diálogo tentativo que está siempre abierto a corrección o completación y que interroga sin violentar, imponer o manipular (Orange, 2011; Sassenfeld, 2010b, 2012a, 2016b; Stern, 2010). Así, el espacio hermenéutico analítico está definido por el esfuerzo continuo de interpretar a través del diálogo entre paciente y terapeuta la experiencia 
subjetiva e intersubjetiva con la finalidad de comprenderla. Un espacio analítico de este tipo se basa en lo que Orange (2011) llama una hermenéutica de la confianza, característica del psicoanálisis relacional e intersubjetivo, que en su opinión contrasta con la hermenéutica de la sospecha propia del psicoanálisis clásico. Quizás la característica principal de una hermenéutica de la confianza es, al menos para Gadamer, la disposición a asumir que el otro puede estar en lo correcto y uno equivocado.

\section{La curvatura del espacio intersubjetivo}

En el contexto de su filosofía fenomenológica y ética, Lévinas (1961) plantea la existencia de una curvatura en el espacio intersubjetivo que daría cuenta de la dimensión ética de la intersubjeividad. ¿Qué puede significar esto? Para Lévinas, la curvatura del espacio intersubjetivo expresa fundamentalmente la forma basal de relación ética entre seres humanos cuando se encuentran uno frente al otro. En su opinión, puede asumirse en el nivel más básico del encuentro humano una asimetría vincular intrínseca en la cual la necesidad y vulnerabilidad del otro -que Lévinas llama el rostro del otro- caracteriza la experiencia vivida del otro. La experiencia del rostro del otro, el rostro del desconocido que sufre (Orange, 2011), nos coloca en un lugar de responsabilidad respecto de ese otro. Esa es, de acuerdo a Lévinas, la experiencia ética originaria que nos comanda a socorrer al otro y que, así, hace del espacio intersubjetivo un espacio asimétrico -contexto en el cual es relevante recordar la definición del espacio analítico como caracterizado por una mutualidad asimétrica (Aron, 1996; Hoffman, 1998; Sassenfeld, 2012a). Al menos por algunos instantes, estoy para ti (Lévinas, 1974; Sassenfeld, 2016b). Esto no implica necesariamente imaginar escenarios extremos, como los que en ocasiones se asocian al pensamiento levinasiano; por lo contrario, un ejemplo que Lévinas trae a colación con relativa frecuencia es la experiencia de encontrarse con alguien desconocido frente a la entrada de un lugar al cual ambos buscamos entrar y estar dispuesto de manera genuina -a diferencia de hacerlo por obligación moral- a dejar pasar al otro antes que pasar uno: "Après vous".

La experiencia relacional que Lévinas busca poner de relieve solo es posible enlazada con la simultánea, aunque momentánea, presencia de una subjetividad mínima en el sujeto que percibe el rostro del otro (Orange, 2011; Sassenfeld, 2016b). En otras palabras, la experiencia del rostro del otro implica una temporal disminución de la relevancia y el predominio de los propios intereses, preocupaciones y deseos. Es probable que a muchos psicoterapeutas no les cueste reconocer la experiencia detallada por Lévinas; por lo común, mientras atendemos pacientes, nuestras preocupaciones personales pasan con cierta facilidad a un segundo plano mientras estamos trabajando, por mucho que existan jornadas 
particulares en las cuales estamos más interferidos por nuestras problemáticas personales. Sin esa subjetividad mínima no es posible percibir el rostro del otro y actuar en concordancia con esa percepción. Ahora bien, lo descrito por Lévinas no debe entenderse como un mandato ético, como algo que es necesario "hacer" -lo cual, de hecho, lo convertiría en un mandato moral. Su filosofía busca, más bien, dar cuenta de una experiencia que surge y se despliega (o no) sin estar mediada por la voluntad o la deliberación; por lo tanto, se trata de una experiencia que muchas veces surge de modo espontáneo y muchas veces no. Una circunstancia que, para Lévinas, no le quita su importancia a la hora de comprender la experiencia ética, sino todo lo contrario.

En el marco del psicoanálisis relacional e intersubjetivo, Orange (2011) ha vinculado las contribuciones de Lévinas con una ética clínica del alivio del sufrimiento. Y, aunque también el psicoterapeuta sufre, en la situación analítica el sufrimiento del paciente es preponderante: el psicoterapeuta se tiende a encontrar en un estado de subjetividad mínima. Siguiendo a Orange, ya el hecho de solicitar atención psicoterapéutica coloca al paciente en un lugar de mayor vulnerabilidad que el terapeuta. Por otro lado, el psicoanálisis relacional e intersubjetivo cuenta entre sus méritos haber concedido mayor importancia y realidad a la subjetividad del psicoterapeuta y a la influencia de ésta en el paciente y en el proceso analítico. En consecuencia, la discusión entre defensores de la necesidad de una subjetividad mínima por parte del terapeuta y la necesidad de que el terapeuta no someta o disocie aspectos centrales de su propia subjetividad frente al predominio más general de la subjetividad del paciente está lejos de estar resuelta satisfactoriamente. Esta controversia puede, en efecto, advertirse hasta el día de hoy con claridad en algunas discusiones entre quienes adhieren a la psicología del self y sus desarrollos post-kohutianos y quienes adhieren a las teorías relacionales más marcadas por los teóricos analíticos con trasfondos formativos ligados al psicoanálisis interpersonal. A mi parecer, es evidente que ambos planteamientos recogen aspectos fundamentales de la práctica analítica contemporánea.

Con todo, quizás la descripción que Lewis Aron (1996) realiza del vínculo analítico como mutuo pero asimétrico es hasta el momento la formulación más afortunada. Subraya la mutualidad existente entre paciente y terapeuta, pero a la vez destaca la existencia de diferencias fundamentales en términos de roles y, sobre todo, de responsabilidades. Que el vínculo analítico siga siendo un vínculo analítico es, antes que nada, responsabilidad del psicoterapeuta (Mitchell, 1997), como también lo es buscar la forma de aliviar el sufrimiento del paciente en alguna medida (Orange, 2011). Se debe a esto que las contribuciones de Lévinas a la comprensión de la naturaleza de la relación analítica hayan tenido una resonancia considerable en la comunidad psicoanalítica relacional e intersubjetiva. 
Comentarios finales

Este trabajo ha buscado dar cuenta de algunas contribuciones esenciales de la filosofía fenomenológica y hermenéutica a una concepción intersubjetiva del vínculo analítico. En este breve recorrido, he enfatizado diferentes elementos complementarios: la relación analítica como vínculo afectivo, como situación analítica, fundamentalmente como espacio hermenéutico $y$, por último, como relación marcada por la ética. Ninguno de estos elementos por separado puede bastar para esbozar una comprensión abarcadora del vínculo analítico y, seguramente, hacen falta numerosos elementos adicionales para delinear tal comprensión. En este contexto, me he limitado a algunos aportes filosóficos particulares que, sin embargo, han sido fundamentales en el surgimiento del psicoanálisis relacional e intersubjetivo. El giro fenomenológico hacia la experiencia vivida y el giro hermenéutico hacia la comprensión a través del diálogo en el psicoanálisis lo han transformado y vitalizado de manera profunda. El enriquecimiento que el psicoanálisis relacional e intersubjetivo ha obtenido y que puede seguir obteniendo de su contacto entusiasta con la filosofía es, a mi parecer, innegable (Sassenfeld, 2016b). Espero haber podido transmitir algo de ese enriquecimiento en este trabajo.

\section{REFERENCIAS}

Angehrn, E. (2004). Interpretation und Dekonstruktion: Untersuchungen zur Hermeneutik. Weilerwist: Velbrück Wissenschaft.

Aron, L. (1996). A Meeting of Minds: Mutuality in Psychoanalysis. New Jersey: The Analytic Press.

Askay, R. \& Farquhar, J. (2006). Apprehending the Inaccesible: Freudian Psychoanalysis and Existential Phenomenology. Evanston: Northwestern University Press.

Atwood, G. \& Stolorow, R. (2014 [1984]). Structures of Subjectivity: Explorations in Psychoanalytic Phenomenology and Contextualism (2. Ed.). New York: Routledge.

Atwood, G. \& Stolorow, R. (1993). Faces in a Cloud: Intersubjectivity in Personality Theory (2. Ed.). New Jersey: Jason Aronson.

Boston Change Process Study Group (BCPSG) (2010). Change in Psychotherapy: A Unifying Paradigm. New York: W. W. Norton.

Bromberg, P. (2006). Awakening the Dreamer: Clinical Journeys. New Jersey: Analytic Press.

Buirski, P. \& Haglund, P. (2001). Making Sense Together: The Intersubjective Approach to Psychotherapy. New Jersey: Jason Aronson.

Coburn, W. (2014). Psychoanalytic Complexity: Clinical Attitudes for Therapeutic Change. New York: Routledge.

Eagle, M. (2011). From Classical to Contemporary Psychoanalysis: A Critique and Integration. New York: Routledge. 
Figal, G. (2006). Gegenständlichkeit: Das Hermeneutische und die Philosophie. Tübingen: Mohr Siebeck.

Freud, S. (1900). Die Traumdeutung. Frankfurt am Main: S. Fischer Verlag.

Gadamer, H.-G. (1958). Die erkenntnistheoretischen Probleme der Geisteswissenschaften. En H.-G. Gadamer, Das Problem des historischen Bewußtseins (pp. 7-15). Tübingen: Mohr Siebeck.

Gadamer, H.-G. (1960). Wahrheit und Methode: Grundzüge einer philosophischen Hermeneutik. Tübingen: Mohr Siebeck.

Gadamer, H.-G. (1969). Hermeneutik. En H.-G. Gadamer, Hermeneutik II: Wahrheit und Methode. Ergänzungen und Register (Gesammelte Werke II) (pp. 425-436). Tübingen: J. C. B. Mohr.

Gallese, V. (2009). Mirror neurons, embodied simulation, and the neural basis of social cognition. Psychoanalytic Dialogues, 19, 519-536.

Grondin, J. (2001b). Was heißt "Sein, das verstanden werden kann, ist Sprache"? En J. Grondin, Von Heidegger zu Gadamer: Unterwegs zur Hermeneutik (pp. 100-105). Darmstadt: Wissenschaftliche Buchgesellschaft.

Heidegger, M. (1982 [1923]). Ontologie (Hermeneutik der Faktizität). Frankfurt am Main: Vittorio Klostermann.

Heidegger, M. (1927). Sein und Zeit. Tübingen: Max Niemeyer Verlag.

Hoffman, I. (1998). Ritual and Spontaneity in the Psychoanalytic Process: A Dialectical-Constructivist View. New York: The Analytic Press.

Husserl, E. (1936). Die Krisis der europäischen Wissenschaften und die transzendentale Phänomenologie: Eine Einleitung in die phänomenologische Philosophie. Hamburg: Felix Meiner Verlag.

Jordán, J. F. (2008). Intersubjetividad: El giro fenomenológico en el psicoanálisis. Revista Chilena de Psicoanálisis, 25 (1), 6-16.

Kohut, H. (1977). The Restoration of the Self. Chicago: The University of Chicago Press.

Kohut, H. (1984). How Does Analysis Cure? Chicago: Chicago University Press.

Leffert, M. (2016). Phenomenology, Uncertainty, and Care in the Therapeutic Encounter. New York: Routledge.

Lévinas, E. (1961). Totalität und Unendlichkeit: Versuch über die Exteriorität. Freiburg/München: Verlag Karl Alber.

Lévinas, E. (1974). Jenseits des Seins oder anders als Sein geschieht. Freiburg/München: Verlag Karl Alber.

Lichtenberg, J., Lachmann, F. \& Fosshage, J. (1996). The Clinical Exchange: Techniques Derived From Self and Motivational Systems. New York: Routledge.

Lichtenberg, J., Lachmann, F. \& Fosshage, J. (2016). Enlivening the Self: The First Year, Clinical Enrichment, and the Wandering Mind. New York: Routledge.

Merleau-Ponty, M. (1945). Phänomenologie der Wahrnehmung. Berlin: Walter de Gruyter.

Mitchell, S. (1993). Hope and Dread in Psychoanalysis. New York: Basic Books.

Mitchell, S. (1997). Psychoanalyse als Dialog: Einfluss und Autonomie in der analytischen Beziehung. Giessen: Psychosozial Verlag.

Orange, D. (1995). Emotionales Verständnis und Intersubjektivität: Beiträge zu einer psychoanalytischen Epistemologie. Frankfurt am Main: Brandes \& Apsel.

Orange, D. (2010). Thinking for Clinicians: Philosophical Resources for Contemporary Psychoanalysis and the Humanistic Psychotherapies. New York: Routledge.

\section{CeIR Vol. 11 (3) - Octubre 2017 ISSN 1988-2939 - www.ceir.info}

(c) Derechos reservados/Copyright de Clínica e investigación Relacional y los autores. Prohibida la reproducción total o parcial sin autorización expresa. Este material es para uso científico y profesional exclusivamente y puede contener información clínica sensible. Los editores no se responsabilizan de los contenidos de los autores. Dirigir las consultas sobre derechos y autorizaciones a ceir@psicoterapiarelacional.es 
Orange, D. (2011). The Suffering Stranger: Hermeneutics for Everyday Clinical Practice. New York: Routledge.

Orange, D., Atwood, G. \& Stolorow, R. (1997). Intersubjektivität in der Psychoanalyse: Kontextualismus in der psychoanalytischen Praxis. Frankfurt am Main: Brandes \& Apsel.

Renik, O. (1993). Analytic interaction: Conceptualizing technique in light of the analyst's irreducible subjectivity. En S. Mitchell \& L. Aron (Eds.), Relational Psychoanalysis: The Emergence of a Tradition (pp. 407-424). New Jersey: The Analytic Press.

Reis, B. (1999). Thomas Ogden's phenomenological turn. Psychoanalytic Dialogues, 9 (3), 371-393.

Rorty, R. (2001). "Sein, das verstanden werden kann, ist Sprache". En "Sein, das verstanden werden kann, ist Sprache" (pp. 30-49). Frankfurt am Main: Edition Suhrkamp.

Safran, J. (2012). Psychoanalysis and Psychoanalytic Therapies. Washington: American Psychological Association.

Sassenfeld, A. (2006). El concepto del selfobjeto y el proceso psicoterapéutico desde la perspectiva de la psicología psicoanalítica del self. Gaceta Universitaria, 2 (1), 55-60.

Sassenfeld, A. (2007). Del cuerpo individual a un cuerpo relacional: Dimensión somática, interacción y cambio en psicoterapia. Gaceta de Psiquiatría Universitaria: Temas y Controversias, 3 (2), 177-188.

Sassenfeld, A. (2008). Lenguaje corporal e intencionalidad relacional. Gaceta de Psiquiatría Universitaria, 4 (1), 83-92.

Sassenfeld, A. (2010a). Fundamentos de la relación psicoterapéutica en la psicoterapia corporal relacional. Gaceta de Psiquiatría Universitaria, 6 (1), 51-62.

Sassenfeld, A. (2010b). Algunas reflexiones sobre la hermenéutica filosófica de Hans-Georg Gadamer y su relación con la práctica de la psicoterapia. Gaceta de Psiquiatría Universitaria, 6 (4), 427-448.

Sassenfeld, A. (2010c). Körpersprache und relationale Intentionalität. Psychoanalyse und Körper, 9 (2), 71-89.

Sassenfeld, A. (2010d). Enactments: Una perspectiva relacional sobre vínculo, acción e inconsciente. Clínica e Investigación Relacional, 4 (1), 142-181.

Sassenfeld, A. (2012a). Principios clínicos de la psicoterapia relacional. Santiago de Chile: SODEPSI Ediciones.

Sassenfeld, A. (2012b). Sistemas intersubjetivos encarnados: De la sugestión a la influencia mutua. Gaceta de Psiquiatría Universitaria, 8 (3), 310-321.

Sassenfeld, A. (2012C). Gegenseitige Beeinflussung und Suggestion aus der Sicht einer verkörperten Intersubjektivitätstheorie. Psychoanalyse und Körper, 11 (2), 9-30.

Sassenfeld, A. (2013a). Verkörperte Intersubjektivität und sensomotorische Aspekte von Organisationsprinzipien der Subjektivität. Psychoanalyse und Körper, 22 (1), 11-42.

Sassenfeld, A. (2013b). Die implizite Domäne: Annäherungen an ihre Definition. En P. Geißler \& A. Sassenfeld (Eds.), Jenseits von Sprache und Denken: Implizite Dimensionen im psychotherapeutischen Geschehen (pp. 57-133). Giessen: Psychosozial Verlag.

Sassenfeld, A. (2013c). Implizites reziprokes Körperlesen. En P. Geißler \& G. Heisterkamp (Eds.), Einführung in die analytische Körperpsychotherapie (pp. 103-107). Giessen: Psychosozial Verlag.

Sassenfeld, A. (2014). La intersubjetividad corporizada y aspectos sensoriomotrices de los principios organizadores de la subjetividad. Clínica e Investigación Relacional, 8 (2), 425-457. 
Sassenfeld, A. (2015). Relational analytical psychotherapy as hermeneutic space. Presentación en XVII International Conference on Philosophy, Psychiatry, and Psychology, "Why do humans become mentally ill? Anthropological, biological, and cultural vulnerabilities of mental illness", Frutillar, Chile.

Sassenfeld, A. (2016a). Algunas contribuciones de la filosofía fenomenológica y hermenéutica a una concepción relacional del vínculo analítico. En R. Pulido \& E. Thumala (Eds.),

Sassenfeld, A. (2016b). El espacio hermenéutico: Comprensión y espacialidad en la psicoterapia analítica intersubjetiva. Santiago de Chile: SODEPSI Ediciones.

Sletvold, J. (2014). The Embodied Analyst: From Freud and Reich to Relationality. New York: Routledge.

Slochower, J. (2014 [2006]). Psychoanalytic Collisions (2. Ed.). New York: Routledge.

Stern, D. B. (1997). Unformulated Experience: From Dissociation to Imagination in Psychoanalysis. New York: Psychology Press.

Stern, D. B. (2010). Partners in Thought: Working with Unformulated Experience, Dissociation, and Enactment. New York: Routledge.

Stern, D. B. (2015). Relational Freedom: Emergent Properties of the Interpersonal Field. New York: Routledge.

Stern, D. N. (2004). The Present Moment in Psychotherapy and Everyday Life. New York: W. W. Norton.

Stolorow, R. (2010). Un analista fenomenológico-contextual: la teoría de los sistemas intersubjetivos y la práctica clínica. Entrevista a Robert Stolorow. Gaceta de Psiquiatría Universitaria, 6 (3), 278-283.

Stolorow, R. (2011). World, Affectivity, Trauma: Heidegger and Post-Cartesian Psychoanalysis. New York: Routledge.

Stolorow, R. \& Atwood, G. (1992). Los contextos del ser: Las bases intersubjetivas de la vida psíquica. Madrid: Herder.

Stolorow, R., Atwood, G. \& Orange, D. (2002). Worlds of Experience: Interweaving Philosophical and Clinical Dimensions in Psychoanalysis. New York: Basic Books.

Stolorow, R., Brandchaft, B. \& Atwood, G. (1987). Psychoanalytische Behandlung: Ein intersubjektiver Ansatz. Frankfurt am Main: Fischer Taschenbuch Verlag.

Summers, F. (2013). The Psychoanalytic Vision: The Experiencing Subject, Transcendence, and the Therapeutic Process. New York: Routledge.

Summers, F. (2015). Los fundamentos del psicoanálisis en la filosofía fenomenológica y la evolución de la teoría psicoanalítica. Clínica e Investigación Relacional, 9 (1), 11-32.

Tauber, A. (2010). Freud, The Reluctant Philosopher. Princeton: Princeton University Press.

Taylor, C. (1985). Self-interpreting animals. En C. Taylor, Philosophical Papers (Vol. 1) (pp. 45-76). Cambridge: Cambridge University Press.

Teicholz, J. (1999). Kohut, Loewald, and the Postmoderns: A Comparative Study of Self and Relationship. New York: Routledge.

Wachtel, P. (2008). Relational Theory and the Practice of Psychotherapy. New York: Guilford Press.

Wachtel, P. (2014). Cyclical Psychodynamics and the Contextual Self: The Inner World, the Intimate World, and the World of Culture and Society. New York: Routledge.

Original recibido con fecha: 19/07/2017 Revisado: 24/07/2017 Aceptado: 30/09/2017

@ ( Derechos reservados/Copyright de Clínica e investigación Relacional y los autores. Prohibida la reproducción total o parcial sin autorización expresa. Este material es para uso científico y profesional exclusivamente y puede contener información clínica sensible. Los editores no se responsabilizan de los contenidos de los autores. Dirigir las consultas sobre derechos y autorizaciones a ceir@psicoterapiarelacional.es 
NOTAS:

${ }^{1}$ El verbo alemán sein contiene ambos significados, ser y estar, para los cuales el español tiene palabras separadas. Trasciende por mucho las limitaciones de este trabajo la elucidación de las implicancias de esta circunstancia.

${ }^{2}$ Recuérdese, en este contexto, que para Gadamer (1960) la noción de prejuicio no tiene una connotación negativa por sí misma; Gadamer buscó, en sus propias palabras, rehabilitar la noción de prejuicio afirmando que nuestro prejuicio puede, cuando actúa de forma inconsciente, interferir en nuestra aprehensión del mundo, las cosas y los otros pero, por otro lado, mostrando que nuestro prejuicio es a la vez lo que posibilita nuestro acceso y nuestra comprensión del mundo, las cosas y los otros (Sassenfeld, 2010b, 2012a, 2016b).

3 Dicho sea de paso que, en mi opinión, en términos generales tanto el psicoanálisis freudiano como el psicoanálisis relacional tienen una deuda con sus concepciones teóricas respecto del lenguaje. Stern (1997, 2010) ha comenzado a pensar sobre este punto. 